\title{
Renal histopathology spectrum in children with kidney diseases in Saudi Arabia, 1998-2017
}

\author{
Khalid Alhasan, MD, Noura M. Aloudah, MD, Amaar A. Bakhit, MRCP UK, SBIM, Yassin M. Alhamad, MBBS, \\ Kechrid M. Chihabeddine, MD, AFSA, Talal A. Alfaadhel, MBBS, FRCPC, Tariq E. Aljohani, MD, KSUFP, \\ Hanadi M. Alhozali, FRCPC, ABIM, Abdulkareem O. Alsuwaida, FRCPC, MSC.
}

\begin{abstract}

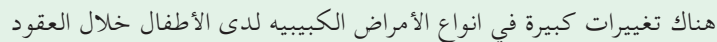

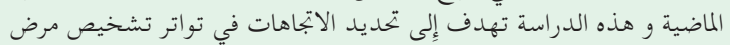

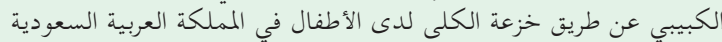

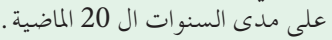

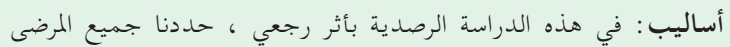

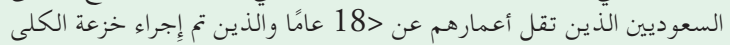

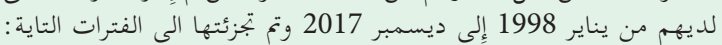

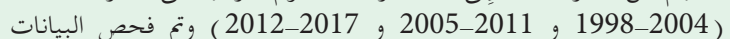
الديمغرافية وارتباطها بانتشار الأمراض الكبيبية المختلفة.

النتائج : تم دراسة 326 حالة مرضية مع خزعة الكلى بمتوسط عمر 11 سنة و

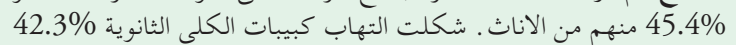

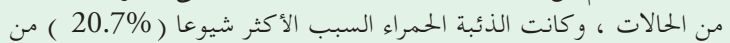

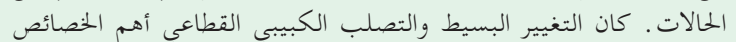

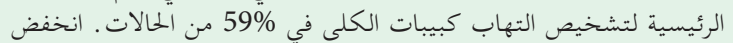

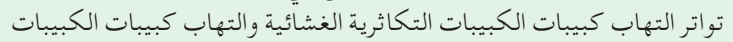

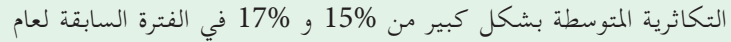

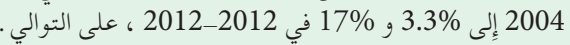

الاستنتاجات : هناك تحولًا كبيرًا في تواتر العديد من الأنواع الفرعية لأمراض

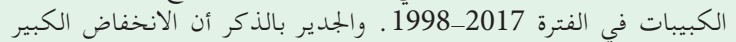

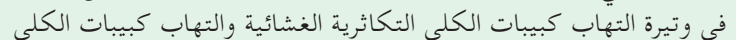

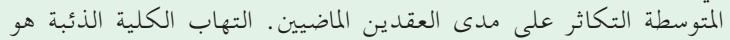
السبب الأكثر شيوعا لالتهاب الكلية الكبيبي الثانوي.
\end{abstract}

Objectives: To identify the trends in the diagnostic frequency of glomerular disease subtypes by renal biopsy in children in Saudi Arabia over the last 20 years.

Methods: In this retrospective observational study, we identified all patients aged $<18$ years for whom native kidney biopsy was performed between 1998 and 2017. The period during which biopsy was performed (1998-2004, 2005-2011, and 2012-2017) and the demographic information and their association with the prevalence of various glomerular disease subtypes were our primary outcomes.
Results: A total of 326 cases with renal biopsy were analyzed; the mean age of participants being 11 years and $45.4 \%$ of them were girls. Unexpectedly, secondary glomerulonephritis accounted for $42.3 \%$ of the cases, and lupus nephritis was the most common cause noted in $20.7 \%$ of the cases. The minimal change and focal segmental glomerulosclerosis were the most common glomerulonephritis in 59\% of the cases. The frequency of membranoproliferative glomerulonephritis and mesangioproliferative glomerulonephritis significantly decreased from $15 \%$ and $17 \%$ in the period prior to 2004 to $3.3 \%$ $(p=0.003)$ and $1.7 \%$ in $2012-2017(p<0.001)$.

Conclusions: We found a considerable shift in the frequency of many glomerular disease subtypes in 1998-2017, which make clinical predication of the underlying etiology challenging for clinician. Renal biopsy still remains a critical diagnostic procedure for managing a considerable proportion of renal diseases.

Keywords: glomerulonephritis, biopsy, nephrotic syndrome, focal segmental glomerulosclerosis

Saudi Med J 2020; Vol. 41 (4): 369-375

doi: 10.15537/smj.2020.4.24999

From the Department of Pediatric (Alhasan), from the Department of Medicine (Bakhit, Alhamad, Alfaadhel), from the Department of Pathology (Aljohani), King Saud University; from the Department of Pathology (Aloudah), King Abdulaziz Medical City; from the Department of Medicine (Chihabeddine), Security Forces Hospital; from the Department of Medicine (Alhozali), King Abdulaziz University Hospital; and from the Department of Medicine (Alsuwaida), King Abdullah Bin Abdulaziz Hospital, Princess Nourah Bint Abdulrahman University, Riyadh, Kingdom of Saudi Arabia.

Received 9th October 2019. Accepted 9th February 2020.

Address correspondence and reprint request to: Dr. Abdulkareem O. Alsuwaida, Department of Medicine, King Abdullah Bin Abdulaziz Hospital, Princess Nourah bint Abdulrahman University, Riyadh, Kingdom of Saudi Arabia. E-mail: aoalsuwaida@kaauh.edu.sa

ORCID ID: https://orcid.org0000-0001-5470-5557 
$\mathrm{R}^{\mathrm{c}}$ enal disease has been recognized as an important cause of morbidity and mortality for the last decades in Saudi Arabia. ${ }^{1,2}$ The main consequences of kidney disease include disease progression and, subsequently, increased risk of cardiovascular disease. Although advances in technology and medical treatment have been reported during the past decades, ${ }^{3,4}$ data on the incidence and prevalence of various types of glomerular diseases in the kingdom have been limited. Additionally, most of the reports are outdated and do not reflect the spectrum of glomerular diseases among Saudi children in the last 10-20 years. In a previous study conducted at King Faisal Specialist Hospital and Research Centre, Riyadh, researchers identified a high proportion of focal segmental glomerulosclerosis (FSGS) and a low proportion of membranous nephropathy (MN) cases among 147 renal biopsy samples. ${ }^{5}$ In another multicenter study conducted in Saudi Arabia, ${ }^{2}$ investigators found that the most common renal diseases were FSGS (34.9\%), mesangioproliferative glomerulonephritis (MesPGN) (29.1\%), and membranoproliferative glomerulonephritis (MPGN) (10.5\%). The least frequent were MN (6.5\%), minimal change disease (MCD) (5.8\%), and immunoglobulin A (IgA) nephropathy (5.8\%). However, both studies included both adult and pediatric cases.

Therefore, this study aimed to examine the trend of various pathologies in native kidney biopsies conducted in children between 1998 and 2017, to describe trends in various glomerular disease frequencies over the last 2 decades, and to examine their relationship with age and gender. We also report the clinical presentation of different glomerular diseases.

Methods. This is a cross-sectional multicenter on native kidney biopsy specimens of glomerular disease conducted in children from January 1998 to December 2017, including 4 tertiary referral hospitals in Saudi Arabia: King Saud University Medical City in Riyadh, Security Forces Hospital in Riyadh, King Abdulaziz Medical City in Riyadh, and King Abdulaziz University Hospital in Jeddah. After excluding patients with missing histologic diagnosis or with inadequate biopsy (adequate biopsy was defined as 10 or more glomeruli), a total of 326 biopsies were included in the analysis. Pediatric patients were divided into 2 age

Disclosure. Authors have no conflict of interests, and the work was not supported or funded by any drug company. strata: young children (aged 0-12 years) and adolescents (aged 13-17 years). The indications for kidney biopsy included nephrotic syndrome with steroid resistant after 6-8 weeks of therapy or frequent relapse, nephritic syndrome, unexplained renal impairment, subnephrotic proteinuria, asymptomatic urine abnormalities. Institutional Review Board (IRB) approval for the study was obtained from King Khalid University Hospital, Riyadh, Saudi Arabia (E12-811), and a waiver of consent was granted.

All renal biopsies had been carried out by either pediatric nephrologist or interventional radiologist under real-time ultrasound guidance. Most of the time, the renal biopsy carried out under general anesthesia specially in children below 12 years of age. All renal biopsy specimens were processed using the standard light, immunofluorescence, and electron microscopy procedures. Diagnoses were made by nephropathologists. Clinical, demographic, and laboratory data and renal histopathological diagnosis were collected from patient's medical records. There were 20 renal pathologists involved in reporting of kidney biopsies.

The histologic findings were classified according to Revised Protocol for the Histological Typing of Glomerulopathy. ${ }^{6}$ Histologic diagnoses were classified into one of 4 major categories: (i) primary glomerulopathy, such as MCD, IgA nephropathy, MGN, MPGN, mesangioproliferative, and FSGS; (ii) secondary glomerulopathy associated with primary diseases, including but not limited to lupus, HenochSchonlein purpura, and diabetes; (iii) hereditary glomerulopathy, such as Alport syndrome; (iv) and unclassified glomerulopathy, such as end-stage kidney disease of undetermined origin.

Timing of biopsy period was our primary exposure, categorized in to 3 consecutive 7-year time intervals (1998-2004, 2005-2011, and 2012-2017) for data analysis. Glomerular disease subtype frequency was our primary outcome. As secondary outcomes, the clinical presentation and trends in glomerular disease frequency within demographic subgroups were examined. Ethical approval was obtained from Ethics Committee of College of Medicine Research Center at King Saud University according to principles of Helsinki Declaration.

Statistical analysis. Statistical analyses were performed using SAS software (SAS Institute, Cary, US). Categorical variables are reported as absolute number and percentage, and the continuous variables are presented as the mean \pm standard deviation. When analyzing different glomerular diseases across the study 
eras, probability for trend values are reported. Differences in the frequency of various glomerular diseases among age groups were compared using the Chi-square test/ Fisher exact test. A $p \leq 0.05$ was considered significant.

Results. Among the 326 cases analyzed, more than half the patients were male. The mean age of the patients at biopsy was $11.0 \pm 5.0$ years. The mean urine protein, serum albumin, and serum creatinine levels are presented in Table 1.

Gender and age characteristics of patients with primary glomerular disease are shown in Table 2. In most cases, male was more commonly affected, except for membranous nephropathy $(\mathrm{MN})$, where both sexes were equally affected.

The frequency of MPGN in 2012-2017 significantly decreased to $3.3 \%$ and MesPGN to $1.7 \%$, compared to that in the period before 2004; the frequency of MPGN in $1998-2004$ were $15 \%(p=0.003)$ and MesPGN were $17.5 \%(p<0.001)$ (Figure 1). Conversely, the frequency of MCD consistently increased across the study periods, from $8.8 \%$ in $1998-2004$ to $18.3 \%$ in $2012-2017$ $(p=0.1)$. The frequencies of $\operatorname{IgA}$ nephropathy $(\operatorname{IgAN})$

Table 1 - Demographic and indication for kidney biopsy of 326 Saudi children who underwent kidney biopsy in from 1998 to 2017.

\begin{tabular}{lr}
\hline Demographic and indication & $\mathbf{n}(\%)$ \\
\hline Gender & $178(54.6)$ \\
Male & $148(45.4)$ \\
Female & 4.0 \\
Urine protein (g/day) & 2.5 \\
Median & 24.3 \\
Serum albumin & $11.0(5.0)$ \\
Age at biopsy, mean in years (SD) & 91 \\
Serum creatinine ( $\mu$ mol/L) & 52 \\
Median ( $\mu$ mol/L) & \\
Indication for kidney biopsy & $171(52.5)$ \\
Nephrotic syndrome & $118(36.2)$ \\
Nephritic syndrome & $9(2.8)$ \\
Unexplained renal impairment & $20(6.1)$ \\
Asymptomatic urinary abnormalities & $8(2.4)$ \\
Subnephrotic proteinuria & \\
Histological diagnosis & $182(55.8)$ \\
Primary glomerular disease & $138(42.3)$ \\
Secondary glomerular disease & $6(1.8)$ \\
Others &
\end{tabular}

Table 2 - Gender and age characteristics of 326 children patients with primary glomerular disease underwent kidney biopsy in Saudi Arabia from 1998 to 2017.

\begin{tabular}{lcrr}
\hline $\begin{array}{l}\text { Glomerular } \\
\text { pattern }\end{array}$ & $\mathbf{n}(\%)$ & $\begin{array}{c}\text { Gender } \\
\text { M:F } \\
(P \text {-value })\end{array}$ & $\begin{array}{c}\text { Age (years) } \\
\text { mean }\end{array}$ \\
\hline FSGS & $56(30.8)$ & $2: 1(0.05)$ & $10.5 \pm 5.6$ \\
MCD & $52(28.6)$ & $1.6: 1 \quad(0.2)$ & $9.5 \pm 5.0$ \\
MN & $13(7.2)$ & $1: 1 \quad(0.5)$ & $14.5 \pm 3.3$ \\
IgAN & $17(9.3)$ & $1.8: 1 \quad(0.3)$ & $11.7 \pm 4.6$ \\
MPGN & $23(12.6)$ & $2.3: 1 \quad(0.1)$ & $9.7 \pm 5.0$ \\
MesPGN & $21(11.5)$ & $2.5: 1 \quad(0.1)$ & $10.4 \pm 5.5$ \\
Total & 182 & & \\
\hline
\end{tabular}

FSGS: focal segmental glomerulosclerosis, MCD: minimal change disease, $\mathrm{MN}$ : membranous nephropathy, IgAN: immunoglobulin A nephropathy, MPGN: membranoproliferative glomerulonephritis, MesPGN: mesangioproliferative glomerulonephritis

and $\mathrm{MN}$ were stable throughout the study periods $(p=0.7$ and $p=0.2)$, whereas the frequency of FSGS modestly increased in 2005-2011 and then slightly decreased in 2012-2017 ( $p=0.7)$.

Secondary glomerulonephritis accounted for 42.3\% of cases (Table 1) and lupus nephritis (LN) was the most common cause, noted in $20.7 \%$ of cases (Figure 2). Minimal change disease and FSGS were the leading primary glomerulonephritis diagnoses, observed in $59 \%$ of cases.

The indications for kidney biopsy varied, with nephrotic syndrome as the most common, followed by unexplained renal impairment (Table 3). As FSGS was the most frequent histological pattern in patients who had renal biopsy for nephrotic syndrome, MPGN was more common among those who had unexplained renal impairment.

When stratified by age group, FSGS was more prevalent among children aged 0-12 years (Table 4). Similarly, MCD was more common in this age group. Conversely, $\mathrm{MN}$ was more common in those aged 13-17 years.

Discussion. Insights into the epidemiology of kidney disease in the pediatric age group provides critical information for pediatric nephrology. Unfortunately, studies investigating the histopathological spectrum of renal biopsies in children are limited, because the most recent available data include all age groups. ${ }^{7,8}$ Additionally, the spectrum of kidney diseases varies in children based on the geographic location. ${ }^{7-11}$ In the 


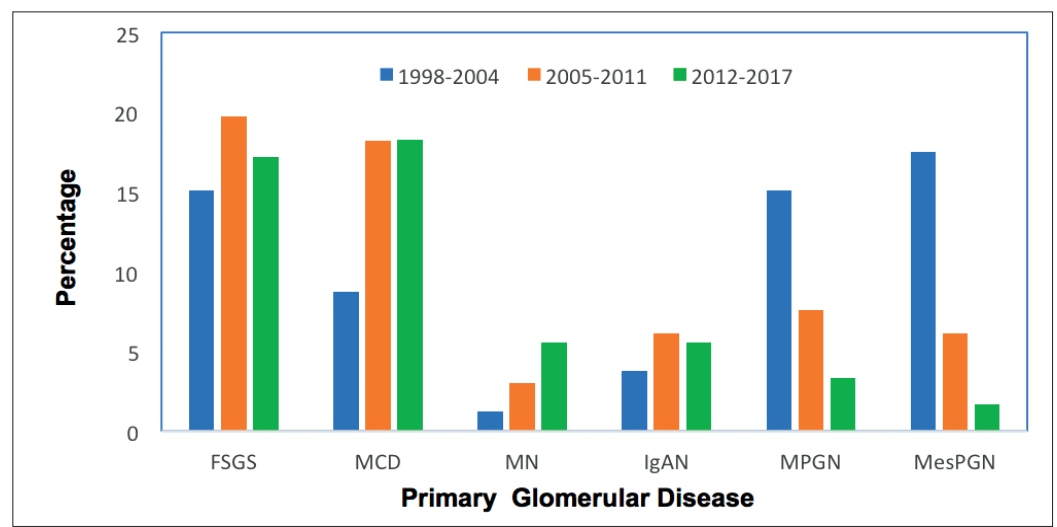

Figure 1 - Temporal trends in primary glomerular disease among 326 children who underwent kidney biopsy in Saudi Arabia from 1998 to 2017. Frequencies of the different subtypes shown as a proportion of the primary glomerular disease cohort. MesPGN: mesangioproliferative glomerulonephritis, MPGN: membranoproliferative glomerulonephritis, IgAN: immunoglobulin A nephropathy, MN: membranous nephropathy, MCD: minimal change disease, FSGS: focal segmental glomerulosclerosis

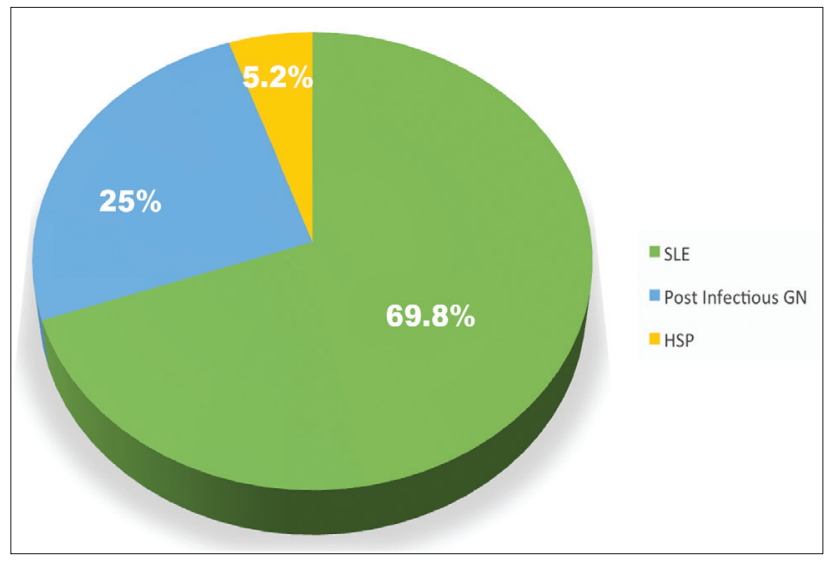

Figure 2 - Frequency of secondary glomerulonephritis among 326 children who underwent kidney biopsy in Saudi Arabia from 1998 to 2017. SLE: systemic lupus erythematosus, GN: glomerulonephritis, HSP: Henoch-Schonlein purpura

study analysis, we examined biopsy results of Saudi children and compared the prevalence of different histopathological subtypes for 3 in 7-year periods. Our analyses indicate a substantial shift in the prevalence of many renal disease subtypes between 1998 and 2017, with an appreciable decline in the prevalence of MPGN and MesPGN between 1998-2004 and 2012-2017. This decline may be due to marked improved socioeconomic conditions and decreased incidence of regional endemic diseases and health care facilities. ${ }^{12,13}$ This finding also in consistent with other studies. ${ }^{14-16}$ However, we cannot exclude the impact of clinician on avoiding doing biopsy in mild cases or those responding to steroid treatment.

In our cohort, the Alport syndrome confirmed by renal biopsy only in 8 patients $(2.5 \%)$. Similar findings have also been reported from other Arab country. More detailed genetic testing is mandated to have the true estimate of secondary cases related to genetic mutations.

As no national data are available on the patterns of diagnosing biopsy-proven renal diseases, findings from international cohorts have shown temporal shifts in the frequency of renal disease subtypes during the past 3 decades. ${ }^{7,8}$ In one report, ${ }^{8}$ investigators found a steady increase in the frequency of diabetic glomerulosclerosis in adolescents over the 3 study decades. Furthermore, they described an initial increase in the frequency of FSGS, which then plateaued and ultimately declined. The frequency of other glomerular subtypes, such as $\mathrm{MN}$ and MCD, considerably increased in all study intervals. In contrast, they observed a relatively moderate decline in the frequency of LN and MPGN, while the frequency of IgA nephropathy and anti-neutrophil cytoplasmic antibodies (ANCA)/pauci-immune glomerulonephritis (GN) remained stable. ${ }^{8}$ Although these data included all age groups, the researchers did not observe striking variations in the frequency of renal disease subtypes among the different age groups. The mean age for patients with MCD was higher than would be anticipated, likely reflecting the clinical practice on doing kidney biopsy in children with nephrotic syndrome.

A more surprising finding was the decreasing 
Table 3 - Indications for kidney biopsy in patients with primary glomerular disease stratified by histological pattern.

\begin{tabular}{|c|c|c|c|c|c|c|}
\hline \multirow[t]{2}{*}{ Clinical presentation } & \multicolumn{6}{|c|}{ Histological pattern } \\
\hline & FSGS & MPGN & MCD & MN & IgAN & MesPGN \\
\hline Nephrotic syndrome & $45(80.4)$ & $10(43.5)$ & $39(83.0)$ & $11(78.6)$ & $7(41.2)$ & $13(61.9)$ \\
\hline Unexplained renal impairment & $8(14.3)$ & $6(26.1)$ & $3(6.4)$ & 0 & $3(17.6)$ & $2(9.5)$ \\
\hline Asymptomatic urinary abnormalities & 0 & 0 & 0 & 0 & $2(11.8)$ & $1 \quad(4.8)$ \\
\hline Nephritic syndrome & $2(3.6)$ & $6(26.1)$ & 0 & $1(7.1)$ & $4(23.5)$ & $5(23.8)$ \\
\hline Subnephrotic range proteinuria & $1(1.8)$ & $1 \quad(4.3)$ & $5(10.6)$ & $2(14.3)$ & $1(5.9)$ & 0 \\
\hline Total & 56 & 23 & 47 & 14 & 17 & 21 \\
\hline
\end{tabular}

FSGS: focal segmental glomerulosclerosis, MPGN: membranoproliferative glomerulonephritis, MCD: minimal change disease, MN: membranous nephropathy, IgAN: immunoglobulin A nephropathy, MesPGN: mesangioproliferative glomerulonephritis

incidence of FSGS in a multicenter hospital-based study conducted across 115 hospitals in China. ${ }^{17}$ In this report, Nie et $\mathrm{al}^{8}$ studied the spectrum of biopsyproven glomerular diseases among children aged more than 11 years and observed a decrease in the frequency of FSGS from $14 \%$ to $4 \%$, based on the results of the biopsies. This is in stark contrast to the findings of other investigators who reported an increasing frequency of FSGS in American children. Studies including adult patients have also shown consistent increases in the prevalence of FSGS over time, which have been attributed to the increasing obesity rates. ${ }^{18}$ In our report, we observed a modest increase in the rates of FSGS in 2005-2011, followed by a slight decline in 2012-2017. As the rates of obesity among Chinese children are lower than in other regions, this is probably not related to the declining frequency of FSGS, ${ }^{17}$ because obesity-related FSGS is very uncommon in children. ${ }^{9}$ Of note, because Nie et $\mathrm{al}^{17}$ reported biopsy numbers as proportions, a true decline in the incidence of FSGS in their cohort may not be reflected.

Although female represented more than half of the patients in our cohort, the frequency of primary renal disease subtypes was higher in male, except for $M N$, which was equally reported in both sexes. Contrary to our results, other researchers observed a predominance of kidney disease subtypes in male patients. ${ }^{10}$ Similar findings were reported by other authors, who found a higher male-to-female ratio among children with renal disease. ${ }^{19-22}$ Our finding that nephrotic syndrome was the most common indication for kidney biopsy is in line with those reported in studies conducted in other developing countries. ${ }^{10,18}$ Conversely, the indications for renal biopsy are different from those reported by investigators in developed countries, with isolated microscopic hematuria as the most common indication. Nevertheless, that evolving biopsy practice patterns and expanding indications for biopsy may largely differ between institutions and might considerably affect the frequency of biopsies performed in children with kidney disease. In this cohort, only 3 cases $(16.6 \%)$ confirmed to have glomerular disease out of cases biopsied because of asymptomatic urine abnormalities

In the Jordanian cohort, ${ }^{10}$ investigators reported that the most common primary glomerular disease in nephrotic children was MCD, which accounted for $27 \%$ of their cases. As expected, MCD and FSGS were the most common histological subtypes in our study. Although MCD, FSGS, and MPGN are reported as the most frequent histological classifications in children with nephrotic syndrome, ${ }^{23}$ differences in biopsy results and the indications for renal biopsy have been reported across studies. Minimal change disease was identified as the most common histopathologic diagnosis for children receiving a biopsy for nephrotic syndrome in our study and in the Chinese cohort ${ }^{17}$ as well as in a North American cohort of children receiving a biopsy for proteinuria. ${ }^{24}$ Additionally, variations in histopathologic subtypes have been reported across age groups. Also Nasar Yousuf Alwahaibi et al ${ }^{25}$ conducted a systematic review and notice same finding.

In this study, FSGS and MCD were more prevalent in children aged 0-12 years. On the other hand, $\mathrm{MN}$ was more common in those aged 13-17 years. In the Nephrotic Syndrome Study Network (NEPTUNE), ${ }^{24}$ MCD accounted for approximately $65 \%$ of children 
aged $0-11$ years and $35 \%$ of children aged 12-18 years. The investigators reported FSGS as the second most frequent histologic subtype in both age groups. In a Chinese cohort, ${ }^{17}$ the authors found that FSGS was less common in children aged 13-18 years. Since these biopsies were performed during different periods, the disparities between these studies might be due to varying availability of diagnostic resources or changing indications for kidney biopsy in children. Similarly, epidemiologic studies of glomerular disease in children are always limited by the clinical practice of classifying nephrotic syndrome by sensitivity to steroids. While this obviously decreases the need for an invasive procedure in many children with steroid sensitive nephrotic syndrome, it significantly underestimates the incidence rates of minimal change disease. Early renal biopsy in all children with features of lupus nephritis is critical to diagnosis and early management of lupus nephritis. Our study revealed that lupus nephritis was the most common secondary cause, noted in $20.7 \%$ of cases. We are aiming to do more analysis on this data to examine the presentation, histological and clinical outcomes of lupus in children with SLE.

Although this study provides preliminary data for the estimation of trends in the frequency of diagnosing renal biopsy glomerular disease in Saudi children, it has limitations that should be discussed. Since we reported biopsy results as proportions, we could not accurately report the incidence rates of the different histologic subtypes. Additionally, our results may be skewed because we included only cases from 4 major tertiary hospitals across Saudi Arabia. It was not feasible to review the samples in central committee to avoid variation in reporting of various glomerular diseases and data related to biopsy complications were not collected. Similarly, the response to steroid and/or relapses were not captured in our analysis.

In conclusion, primary glomerular disease remains the predominant kidney disease in Saudi children, with MCD and FSGS being the most common pathologies. We found a considerable shift in the frequency of many glomerular disease subtypes in the period 1998-2017. Of note, a considerable decline in the frequency of MPGN and MesPGN over the past 2 decades might reflect improvements in the socioeconomic conditions in the Kingdom. Nevertheless, the underlying reasons for these changing trends should be in future studies to provide new insights into disease pathogenesis or therapeutic options.
Acknowledgment. We would like to thank the Editage (www. Editage.com) for English language editing.

\section{References}

1. Hassanien AA, Al-Shaikh F, Vamos EP, Yadegarfar G, Majeed A. Epidemiology of end-stage renal disease in the countries of the Gulf Cooperation Council: a systematic review. JRSM Short Rep 2012; 3: 38.

2. Akhtar M, Qunibi W, Taher S, Ginn E, Furayh O, Sanjad S, et al. Spectrum of renal disease in Saudi Arabia. Ann Saudi Med 1990; 10: 37-44.

3. Dialysis in the Kingdom of Saudi Arabia. Saudi J Kidney Dis Transplant 2016; 27: 843.

4. Hassanien A. Renal care in Saudi Arabia: A review of the quality of healthcare management. London (UK): Imperial College London; 2018. Available from: http://spiral.imperial.ac.uk/ handle/10044/1/24377

5. Gunibi W, Al-Sibai B, Taher S, Akhtar M. Renal disease in Saudi Arabia: a study of 147 renal biopsies. King Faisal Spec Hosp J 1984; 4: 17-23.

6. Churg J, Bernstein J, Glassock RJ. Renal disease: Classification and Atlas of Glomerular Diseases, 2nd ed. New York (NY): Igaku-Shoin Medical Publishers; 1995. p. 151-178.

7. Hou JH, Zhu HX, Zhou ML, Le WB, Zeng CH, Liang SS, et al. Changes in the spectrum of kidney diseases: An analysis of 40,759 biopsy-proven cases from 2003 to 2014 in China. Kidney Dis 2012; 4: 10-19.

8. O'Shaughnessy MM, Hogan SL, Poulton CJ, Falk RJ, Singh HK, Nickeleit V, et al. Temporal and demographic trends in glomerular disease epidemiology in the Southeastern United States, 1986-2015. Clin J Am Soc Nephrol CJASN 2017; 12: 614-623.

9. Rheault M, Wenderfer S. Evolving epidemiology of pediatric glomerular disease. Clin J Am Soc Nephrol 2018; 13: 977-978.

10. Hadidi R, Hadidi M, alDabbas M. Spectrum of biopsy-proven kidney disease in children at a Jordanian Hospital. Saudi J Kidney Dis Transplant 2014; 25: 680.

11. Abdullah LS. Histopathological pattern of pediatric renal diseases: A study from a university hospital in western Saudi Arabia. Saudi J Kidney Dis Transplant 2012; 23: 377.

12. World Health Organization. WHO EMRO | Infectious disease outbreaks reported in the Eastern Mediterranean Region in 2016. [cited 2018 Aug 17]. Available from: http://www. emro.who.int/fr/surveillance-prevision-et-action/surveillanceinfocus/infectious-disease-outbreaks-reported-in-the-easternmediterranean-region-in-2016.html

13. Abdo AA, Sanai FM, Al-Faleh FZ. Epidemiology of viral hepatitis in Saudi Arabia: Are we off the hook? Saudi J Gastroenterol 2012; 18: 349-357.

14. Volovăt C, Cãruntu I, Costin C, Stefan A, Popa R, Volovăt S, et al. Changes in the histological spectrum of glomerular diseases in the past 16 years in the North-Eastern region of Romania. BMC Nephrol 2013; 15: 148.

15. Farah RI. Glomerulonephritis pattern at a Jordanian Tertiary Care center. Int J Nephrol 2018; 11; 2751372.

16. Al-Homrany M, Alghamdi S, Al-Hwiesh A, Mousa D, Alwakeel J, Mitwalli A, et al. Pattern of renal diseases and the need for establishment of renal biopsy registry in Saudi Arabia. Saudi J Kidney Dis Transpl 2019; 30: 628-633. 
17. Nie S, He W, Huang T, Liu D, Wang G, Geng J, et al. The spectrum of biopsy-proven glomerular diseases among children in China: A national, cross-sectional survey. Clin J Am Soc Nephrol 2018; 13: 1047-1054.

18. Bose B, Cattran D. Glomerular Diseases: FSGS. Clin J Am Soc Nephrol 2014; 9: 626-632.

19. Yadav SP, Shah GS, Mishra OP, Baral N. Pattern of renal diseases in children: A developing country experience. Saudi J Kidney Dis Transpl 2016; 27: 371-376.

20. Becherucci F, Roperto RM, Materassi M, Romagnani P. Chronic kidney disease in children. Clin Kidney J 2016; 9: 583-591.

21. Ladapo TA, Esezobor CI, Lesi FE. Pediatric kidney diseases in an African country: Prevalence, spectrum and outcome. SaudiJ Kidney Dis Transplant 2014; 25: 1110.
22. Harambat J, van Stralen KJ, Kim JJ, Tizard EJ. Epidemiology of chronic kidney disease in children. Pediatr Nephrol Berl Ger 2012; 27: 363-373.

23. Downie ML, Gallibois C, Parekh RS, Noone DG. Nephrotic syndrome in infants and children: pathophysiology and management. Paediatr Int Child Health 2017; 37: 248-258.

24. Gipson DS, Troost JP, Lafayette RA, Hladunewich MA, Trachtman H, Gadegbeku CA, et al. Complete Remission in the Nephrotic Syndrome Study Network. Clin J Am Soc Nephrol CJASN 2016; 11: 81-89.

25. Alwahaibi NY, Al Issaei HK, Al Dhahli BS. Incidence of pediatric glomerular diseases in Arab world: A systematic review. Saudi J Kidney Dis Transpl 2019; 30: 15-23.

\section{Illustrations, Figures, Photographs}

All figures or photographs should be submitted in a high resolution (minimum 300 DPI) electronic version saved in jpeg or tiff format. Original hard copies of all figures may be requested when necessary. Photographs will be accepted at the discretion of the Editorial Board. All lettering, arrows, or other artwork must be done by an artist or draftsman. If arrows are used please ensure they appear in a different color to the background color, preferably black with a white border, or white with a black border. If arrows distinguish different items on the figure then different arrow styles should be used ie. long, short, wide, narrow. Written informed consent for publication must accompany any photograph in which the subject can be identified. Written copyright permission, from the publishers, must accompany any illustration that has been previously published. 